\title{
The large diffusion limit for the heat equation with a dynamical boundary condition
}

\author{
Marek Fila \\ Department of Applied Mathematics and Statistics \\ Comenius University, \\ 84248 Bratislava, Slovakia \\ Kazuhiro Ishige \\ Graduate School of Mathematical Sciences \\ The University of Tokyo \\ 3-8-1 Komaba, Meguro-ku, Tokyo 153-8914, Japan \\ Tatsuki Kawakami \\ Department of Applied Mathematics and Informatics, \\ Ryukoku University \\ Seta Otsu 520-2194, Japan
}

\begin{abstract}
We study the heat equation on a half-space with a linear dynamical boundary condition. Our main aim is to show that, if the diffusion coefficient tends to infinity, then the solutions converge (in a suitable sense) to solutions of the Laplace equation with the same dynamical boundary condition.
\end{abstract}

Keywords: heat equation, dynamical boundary condition, large diffusion limit

\section{Introduction}

We consider the problem

$$
\begin{cases}\varepsilon \partial_{t} u_{\varepsilon}-\Delta u_{\varepsilon}=0, & x \in \mathbb{R}_{+}^{N}, t>0, \\ \partial_{t} u_{\varepsilon}+\partial_{\nu} u_{\varepsilon}=0, & x \in \partial \mathbb{R}_{+}^{N}, t>0, \\ u_{\varepsilon}(x, 0)=\varphi(x), & x \in \mathbb{R}_{+}^{N}, \\ u_{\varepsilon}(x, 0)=\varphi_{b}\left(x^{\prime}\right), & x=\left(x^{\prime}, 0\right) \in \partial \mathbb{R}_{+}^{N},\end{cases}
$$

where $N \geq 2, \mathbb{R}_{+}^{N}:=\mathbb{R}^{N-1} \times \mathbb{R}_{+}, \Delta$ is the $N$-dimensional Laplacian (in $x$ ), $\partial_{t}:=\partial / \partial t, \partial_{\nu}:=$ $-\partial / \partial x_{N}, \varepsilon \in(0,1)$ and $\varphi$ and $\varphi_{b}$ are measurable functions in $\mathbb{R}_{+}^{N}$ and $\mathbb{R}^{N-1}$, respectively. 
Our main aim is to show that, as $\varepsilon \rightarrow 0$, it holds that $u_{\varepsilon} \rightarrow u$ (in a suitable sense), where $u$ is the solution of

$$
\begin{cases}\Delta u=0, & x \in \mathbb{R}_{+}^{N}, t>0, \\ \partial_{t} u+\partial_{\nu} u=0, & x \in \partial \mathbb{R}_{+}^{N}, t>0, \\ u(x, 0)=\varphi_{b}\left(x^{\prime}\right), & x=\left(x^{\prime}, 0\right) \in \partial \mathbb{R}_{+}^{N} .\end{cases}
$$

This convergence does not look unexpected, see [1, but we are not aware of any previous result which would support this natural conjecture. In particular, convergence of this type means that the influence of the initial function $\varphi$ is lost in the limit, and we shall describe this phenomenon in more detail.

A result in a similar spirit was established in [1] for the eikonal equation with the same dynamical boundary condition as in (1.1). More precisely, the following problem was considered in [1]:

$$
\begin{cases}\varepsilon \partial_{t} u_{\varepsilon}+\left|\nabla_{x} u_{\varepsilon}\right|=1, & x \in \Omega, t>0, \\ \partial_{t} u_{\varepsilon}+\partial_{\nu} u_{\varepsilon}=0, & x \in \partial \Omega, t>0, \\ u_{\varepsilon}(x, 0)=\varphi(x), & x \in \bar{\Omega},\end{cases}
$$

where $\varepsilon \in(0,1), \Omega \subset \mathbb{R}^{N}$ is a bounded domain with $C^{1}$-boundary, and $\nu$ is the outer normal of $\partial \Omega$. It was shown in [1] that $u_{\varepsilon} \rightarrow u$ as $\varepsilon \rightarrow 0$, where $u$ is the solution of

$$
\begin{cases}\left|\nabla_{x} u\right|=1, & x \in \Omega, \quad t>0 \\ \partial_{t} u+\partial_{\nu} u=0, & x \in \partial \Omega, \quad t>0\end{cases}
$$

with an appropriate initial condition.

In the context of diffusion, the boundary condition from (1.1) describes thermal contact with a perfect conductor or diffusion of solute from a well-stirred fluid or vapour (see e.g. [8]). Various aspects of analysis of parabolic and elliptic equations with dynamical boundary conditions have been treated by many authors, see for example [2]-77, [9, 11, 12, 21, 22, 25, 27, 29], 33]- 35] for the parabolic case and [10], 13]-[20], 26], 29]-32], 36, 37] for the elliptic one. Here we demonstrate on the simplest possible linear example how are these two classes of problems linked.

Throughout this paper we often identify $\mathbb{R}^{N-1}$ with $\partial \mathbb{R}_{+}^{N}$. We introduce some notation. Let $\Gamma_{D}=\Gamma_{D}(x, y, t)$ be the Dirichlet heat kernel on $\mathbb{R}_{+}^{N}$, that is,

$$
\Gamma_{D}(x, y, t):=(4 \pi t)^{-\frac{N}{2}}\left[\exp \left(-\frac{|x-y|^{2}}{4 t}\right)-\exp \left(-\frac{\left|x-y_{*}\right|^{2}}{4 t}\right)\right]
$$

for $(x, y, t) \in \overline{\mathbb{R}_{+}^{N}} \times \mathbb{R}_{+}^{N} \times(0, \infty)$, where $y_{*}=\left(y^{\prime},-y_{N}\right)$ for $y=\left(y^{\prime}, y_{N}\right) \in \mathbb{R}_{+}^{N}$. Define

$$
\left[S_{1}(t) \phi\right](x):=\int_{\mathbb{R}_{+}^{N}} \Gamma_{D}(x, y, t) \phi(y) d y, \quad(x, t) \in \overline{\mathbb{R}_{+}^{N}} \times(0, \infty),
$$

for any measurable function $\phi$ in $\mathbb{R}_{+}^{N}$. For $x=\left(x^{\prime}, x_{N}\right) \in \overline{\mathbb{R}_{+}^{N}}$ and $t>0$, set

$$
P\left(x^{\prime}, x_{N}, t\right):=c_{N}\left(x_{N}+t\right)^{1-N}\left(1+\left|\frac{x^{\prime}}{x_{N}+t}\right|^{2}\right)^{-\frac{N}{2}},
$$


where $c_{N}$ is a constant chosen so that

$$
\int_{\mathbb{R}^{N-1}} P\left(x^{\prime}, x_{N}, t\right) d x^{\prime}=1 \quad \text { for all } x_{N} \geq 0 \text { and } t>0 .
$$

Then $P=P\left(x^{\prime}, x_{N}, t\right)$ is the fundamental solution of the Laplace equation in $\mathbb{R}_{+}^{N}$ with the homogeneous dynamical boundary condition, that is, $P$ satisfies

$$
\begin{cases}-\Delta P=0, & x \in \mathbb{R}_{+}^{N}, t>0, \\ \partial_{t} P+\partial_{\nu} P=0, & x \in \partial \mathbb{R}_{+}^{N}, t>0, \\ P(x, 0)=\delta\left(x^{\prime}\right), & x=\left(x^{\prime}, 0\right) \in \partial \mathbb{R}_{+}^{N},\end{cases}
$$

where $\delta=\delta(\cdot)$ is the Dirac delta function on $\partial \mathbb{R}_{+}^{N}=\mathbb{R}^{N-1}$. Define

$$
\left[S_{2}(t) \psi\right](x):=\int_{\mathbb{R}^{N-1}} P\left(x^{\prime}-y^{\prime}, x_{N}, t\right) \psi\left(y^{\prime}\right) d y^{\prime}, \quad(x, t) \in \overline{\mathbb{R}_{+}^{N}} \times(0, \infty),
$$

for any measurable function $\psi$ in $\mathbb{R}^{N-1}$.

We formulate the definition of a solution of (1.1) by the use of the two integral kernels $\Gamma_{D}$ and $P$. For simplicity, let $\varphi_{b}=\varphi_{b}\left(x^{\prime}\right)$ and $g=g\left(x^{\prime}, t\right)$ be continuous functions in $\mathbb{R}^{N-1}$ and $\mathbb{R}^{N-1} \times(0, \infty)$, respectively, such that $\varphi_{b}\left(x^{\prime}\right)$ and $g\left(x^{\prime}, t\right)$ decay rapidly as $\left|x^{\prime}\right| \rightarrow \infty$. Then the function

$$
w(x, t)=w\left(x^{\prime}, x_{N}, t\right):=\left[S_{2}(t) \varphi_{b}\right](x)+\int_{0}^{t}\left[S_{2}(t-s) g(s)\right](x) d s
$$

can be defined for $x=\left(x^{\prime}, x_{N}\right) \in \mathbb{R}_{+}^{N}$ and $t>0$ and it is a classical solution of the Cauchy problem for the Laplace equation with a nonhomogeneous dynamical boundary condition

$$
\begin{cases}-\Delta w=0, & x \in \mathbb{R}_{+}^{N}, \quad t>0, \\ \partial_{t} w+\partial_{\nu} w=g, & x \in \partial \mathbb{R}_{+}^{N}, \quad t>0, \\ w(x, 0)=\varphi_{b}\left(x^{\prime}\right), & x=\left(x^{\prime}, 0\right) \in \partial \mathbb{R}_{+}^{N} .\end{cases}
$$

It follows from (1.6) that

$$
\begin{aligned}
\partial_{t} w(x, t):= & \int_{\mathbb{R}^{N-1}} \partial_{t} P\left(x^{\prime}-y^{\prime}, x_{N}, t\right) \varphi_{b}\left(y^{\prime}\right) d y^{\prime} \\
& +\int_{\mathbb{R}^{N-1}} P\left(x^{\prime}-y^{\prime}, x_{N}, 0\right) g\left(y^{\prime}, t\right) d y^{\prime} \\
& +\int_{0}^{t} \int_{\mathbb{R}^{N-1}} \partial_{t} P\left(x^{\prime}-y^{\prime}, x_{N}, t-s\right) g\left(y^{\prime}, s\right) d y^{\prime} d s
\end{aligned}
$$

for $x=\left(x^{\prime}, x_{N}\right) \in \mathbb{R}_{+}^{N}$ and $t \in(0, T)$. Set

$$
\Phi(x):=\varphi(x)-\left[S_{2}(0) \varphi_{b}\right](x) .
$$


Then the function

$$
\left.v_{\varepsilon}(x, t):=\left[S_{1}\left(\varepsilon^{-1} t\right) \Phi\right](x)-\int_{0}^{t}\left[S_{1}\left(\varepsilon^{-1}(t-s)\right)\right] \partial_{t} w(s)\right](x) d s
$$

satisfies

$$
\begin{cases}\varepsilon \partial_{t} v_{\varepsilon}=\Delta v_{\varepsilon}-\varepsilon \partial_{t} w, & x \in \mathbb{R}_{+}^{N}, \quad t>0, \\ v_{\varepsilon}=0, & x \in \partial \mathbb{R}_{+}^{N}, \quad t>0, \\ v_{\varepsilon}(x, 0)=\Phi(x), & x \in \mathbb{R}_{+}^{N} .\end{cases}
$$

Let $\partial_{x_{N}}:=\partial / \partial x_{N}$. If $g_{\varepsilon}\left(x^{\prime}, t\right):=\partial_{x_{N}} v_{\varepsilon}\left(x^{\prime}, 0, t\right)$ for $x^{\prime} \in \mathbb{R}^{N-1}, t>0$, and $w_{\varepsilon}$ is defined as in (1.6) with $g_{\varepsilon}$ instead of $g$, then it follows from (1.7), (1.8) and (1.10) that $v_{\varepsilon}$ and $w_{\varepsilon}$ satisfy

$$
\begin{cases}\varepsilon \partial_{t} v_{\varepsilon}=\Delta v_{\varepsilon}-\varepsilon F_{1}\left[\varphi_{b}\right]-\varepsilon F_{2}\left[v_{\varepsilon}\right], & x \in \mathbb{R}_{+}^{N}, t>0, \\ \Delta w_{\varepsilon}=0, & x \in \mathbb{R}_{+}^{N}, t>0, \\ v_{\varepsilon}=0, \quad \partial_{t} w_{\varepsilon}-\partial_{x_{N}} w_{\varepsilon}=\partial_{x_{N}} v_{\varepsilon}, & x \in \partial \mathbb{R}_{+}^{N}, t>0, \\ v_{\varepsilon}(x, 0)=\Phi(x), & x \in \mathbb{R}_{+}^{N}, \\ w_{\varepsilon}(x, 0)=\varphi_{b}\left(x^{\prime}\right), & x=\left(x^{\prime}, 0\right) \in \partial \mathbb{R}_{+}^{N},\end{cases}
$$

where

$$
\begin{aligned}
F_{1}\left[\varphi_{b}\right](x, t):= & \int_{\mathbb{R}^{N-1}} \partial_{t} P\left(x^{\prime}-y^{\prime}, x_{N}, t\right) \varphi_{b}\left(y^{\prime}\right) d y^{\prime}, \\
F_{2}[v](x, t):= & \int_{\mathbb{R}^{N-1}} P\left(x^{\prime}-y^{\prime}, x_{N}, 0\right) \partial_{x_{N}} v\left(y^{\prime}, 0, t\right) d y^{\prime} \\
& +\int_{0}^{t} \int_{\mathbb{R}^{N-1}} \partial_{t} P\left(x^{\prime}-y^{\prime}, x_{N}, t-s\right) \partial_{x_{N}} v\left(y^{\prime}, 0, s\right) d y^{\prime} d s .
\end{aligned}
$$

Furthermore, the function $u_{\varepsilon}:=v_{\varepsilon}+w_{\varepsilon}$ is a classical solution of (1.1). Motivated by this observation, we formulate the definition of a solution of (1.1) via problem (1.11).

Definition 1.1 Let $\varphi$ and $\varphi_{b}$ be measurable functions in $\mathbb{R}_{+}^{N}$ and $\mathbb{R}^{N-1}$, respectively. Let $0<T \leq \infty$ and

$$
v_{\varepsilon}, \partial_{x_{N}} v_{\varepsilon}, w_{\varepsilon} \in C\left(\overline{\mathbb{R}_{+}^{N}} \times(0, T)\right) .
$$

We call $\left(v_{\varepsilon}, w_{\varepsilon}\right)$ a solution of (1.11) in $\mathbb{R}_{+}^{N} \times(0, T)$ if $v_{\varepsilon}$ and $w_{\varepsilon}$ satisfy

$$
\begin{gathered}
v_{\varepsilon}(x, t)=\left[S_{1}\left(\varepsilon^{-1} t\right) \Phi\right](x)-\int_{0}^{t}\left[S_{1}\left(\varepsilon^{-1}(t-s)\right) F_{1}\left[\varphi_{b}\right](s)\right](x) d s \\
-\int_{0}^{t}\left[S_{1}\left(\varepsilon^{-1}(t-s)\right) F_{2}\left[v_{\varepsilon}\right](s)\right](x) d s, \\
w_{\varepsilon}(x, t)=\left[S_{2}(t) \varphi_{b}\right](x)+\int_{0}^{t}\left[S_{2}(t-s) \partial_{x_{N}} v_{\varepsilon}(s)\right](x) d s,
\end{gathered}
$$

for $x \in \overline{\mathbb{R}_{+}^{N}}$ and $t \in(0, T)$. In the case when $T=\infty$, we call $\left(v_{\varepsilon}, w_{\varepsilon}\right)$ a global-in-time solution of (1.11) and $u_{\varepsilon}$ a global-in-time solution of (1.1). 
We are ready to state the main results of this paper. For $1 \leq r \leq \infty$, we write $|\cdot|_{L^{r}}:=$ $\|\cdot\|_{L^{r}\left(\partial \mathbb{R}_{+}^{N}\right)}$ and $\|\cdot\|_{L^{r}}:=\|\cdot\|_{L^{r}\left(\mathbb{R}_{+}^{N}\right)}$ for simplicity.

Theorem 1.1 Let $N \geq 2, \varepsilon \in(0,1), \varphi \in L^{\infty}\left(\mathbb{R}_{+}^{N}\right)$ and $\varphi_{b} \in L^{\infty}\left(\mathbb{R}^{N-1}\right)$. Then problem (1.11) possesses a unique global-in-time solution $\left(v_{\varepsilon}, w_{\varepsilon}\right)$ satisfying

$$
\sup _{0<t<T}\left[\left\|v_{\varepsilon}(t)\right\|_{L^{\infty}}+\left(\varepsilon^{-1} t\right)^{\frac{1}{2}}\left\|\partial_{x_{N}} v_{\varepsilon}(t)\right\|_{L^{\infty}}+\left\|w_{\varepsilon}(t)\right\|_{L^{\infty}}\right]<\infty
$$

for any $T>0$. Furthermore, $v_{\varepsilon}$ and $w_{\varepsilon}$ are bounded and smooth in $\overline{\mathbb{R}_{+}^{N}} \times I$ for any bounded interval $I \subset(0, \infty)$ and have the following properties for any $\tau>0$ :

(a) There exists $C(\tau)>0$ such that

$$
\begin{array}{r}
\sup _{0<t<\tau}\left[\left\|v_{\varepsilon}(t)\right\|_{L^{\infty}}+\left(\varepsilon^{-1} t\right)^{\frac{1}{2}}\left\|\partial_{x_{N}} v_{\varepsilon}(t)\right\|_{L^{\infty}}+\left\|w_{\varepsilon}(t)\right\|_{L^{\infty}}\right] \\
\leq C_{\tau}\left(\|\varphi\|_{L^{\infty}}+\left|\varphi_{b}\right|_{L^{\infty}}\right)
\end{array}
$$

(b) $\lim _{\varepsilon \rightarrow 0} \sup _{0<t<\tau} t^{\frac{1}{2}}\left\|v_{\varepsilon}(t)\right\|_{L^{\infty}\left(\mathbb{R}^{N-1} \times(0, L)\right)}=0$ for any $L>0$;

(c) $\lim _{\varepsilon \rightarrow 0} \sup _{0<t<\tau}\left\|w_{\varepsilon}(t)-S_{2}(t) \varphi_{b}\right\|_{L^{\infty}}=0$.

As a corollary of Theorem 1.1, we see that the solution $u_{\varepsilon}=v_{\varepsilon}+w_{\varepsilon}$ of (1.1) converges to the solution $S_{2}(t) \varphi_{b}$ of $(1.2)$.

Corollary 1.1 Assume the same conditions as in Theorem 1.1. Let $\left(v_{\varepsilon}, w_{\varepsilon}\right)$ be the solution given in Theorem 1.1. Then $u_{\varepsilon}=v_{\varepsilon}+w_{\varepsilon}$ is a classical solution of (1.1) and it satisfies

$$
\lim _{\varepsilon \rightarrow 0} \sup _{\tau_{1}<t<\tau_{2}}\left\|u_{\varepsilon}(t)-S_{2}(t) \varphi_{b}\right\|_{L^{\infty}(K)}=0
$$

for any compact set $K$ in $\overline{\mathbb{R}_{+}^{N}}$ and $0<\tau_{1}<\tau_{2}<\infty$.

We prepare some useful lemmata in Section 2 and then we give a proof of Theorem 1.1 in Section 3 .

\section{Preliminaries}

In this section we prove several lemmata on $S_{1}(t) \phi, F_{1}\left[\varphi_{b}\right]$ and $F_{2}[v]$. In what follows, by the letter $C$ we denote generic positive constants (independent of $x$ and $t$ ) and they may have different values also within the same line.

We first recall the following properties of $S_{1}(t) \phi$ (see e.g. [24]). 
$\left(\mathrm{G}_{1}\right)$ For any $1 \leq q \leq r \leq \infty$

$$
\left\|S_{1}(t) \phi\right\|_{L^{r}} \leq C t^{-\frac{N}{2}\left(\frac{1}{q}-\frac{1}{r}\right)}\|\phi\|_{L^{q}}, \quad t>0
$$

for all $\phi \in L^{q}\left(\mathbb{R}_{+}^{N}\right)$. In particular, if $q=r$, then

$$
\sup _{t>0}\left\|S_{1}(t) \phi\right\|_{L^{r}} \leq\|\phi\|_{L^{r}}
$$

$\left(\mathrm{G}_{2}\right)$ Let $\phi \in L^{q}\left(\mathbb{R}_{+}^{N}\right)$ with $1 \leq q \leq \infty$. Then, for any $T>0, S_{1}(t) \phi$ is bounded and smooth in $\overline{\mathbb{R}_{+}^{N}} \times(T, \infty)$.

Furthermore, we have:

Lemma 2.1 Let $\phi \in L^{\infty}\left(\mathbb{R}_{+}^{N}\right)$. Then

$$
\sup _{t>0} t^{\frac{1}{2}}\left\|\partial_{x_{N}}\left[S_{1}(t) \phi\right]\right\|_{L^{\infty}} \leq\|\phi\|_{L^{\infty}} .
$$

Furthermore,

$$
\limsup _{\varepsilon \rightarrow 0} t_{t>0}^{\frac{1}{2}}\left\|S_{1}\left(\varepsilon^{-1} t\right) \phi\right\|_{L^{\infty}\left(\mathbb{R}^{N-1} \times(0, L)\right)}=0 \quad \text { for any } L>0 .
$$

Proof. It follows from (1.3) that

$$
\begin{aligned}
& K(x, y, t):=\partial_{x_{N}} \Gamma_{D}(x, y, t) \\
& =\Gamma_{N-1}\left(x^{\prime}-y^{\prime}, t\right) \times \\
& \quad \times\left(-\frac{x_{N}-y_{N}}{2 t} \Gamma_{1}\left(x_{N}-y_{N}, t\right)+\frac{x_{N}+y_{N}}{2 t} \Gamma_{1}\left(x_{N}+y_{N}, t\right)\right)
\end{aligned}
$$

for $(x, y, t) \in \overline{\mathbb{R}_{+}^{N}} \times \mathbb{R}_{+}^{N} \times(0, \infty)$, where $\Gamma_{d}(d=1,2, \ldots)$ is the Gauss kernel in $\mathbb{R}^{d}$. Then

$$
\begin{aligned}
& \int_{\mathbb{R}_{+}^{N}}|K(x, y, t)| d y \\
& \leq \int_{0}^{\infty}\left(\frac{\left|x_{N}-y_{N}\right|}{2 t} \Gamma_{1}\left(x_{N}-y_{N}, t\right)+\frac{x_{N}+y_{N}}{2 t} \Gamma_{1}\left(x_{N}+y_{N}, t\right)\right) d y_{N} \\
& =(\pi t)^{-\frac{1}{2}} \int_{0}^{\infty} 2 \eta e^{-\eta^{2}} d \eta=(\pi t)^{-\frac{1}{2}}
\end{aligned}
$$

for $x \in \overline{\mathbb{R}_{+}^{N}}$ and $t>0$. By (1.4) and (2.5) we have

$$
\left|\partial_{x_{N}}\left[S_{1}(t) \phi\right](x)\right| \leq \int_{\mathbb{R}_{+}^{N}}\left|K(x, y, t)\left\|\phi(y) \mid d y \leq t^{-\frac{1}{2}}\right\| \phi \|_{L^{\infty}}\right.
$$

for $x \in \overline{\mathbb{R}_{+}^{N}}$ and $t>0$. This implies (2.2). 
On the other hand, for any $L>0$, it follows from (1.3) that

$$
\begin{aligned}
& \int_{\mathbb{R}_{+}^{N}} \Gamma_{D}\left(x, y, \varepsilon^{-1} t\right) d y \\
& =\int_{0}^{\infty}\left(\Gamma_{1}\left(x_{N}-y_{N}, \varepsilon^{-1} t\right)-\Gamma_{1}\left(x_{N}+y_{N}, \varepsilon^{-1} t\right)\right) d y_{N} \\
& =2\left(4 \pi \varepsilon^{-1} t\right)^{-\frac{1}{2}} \int_{0}^{x_{N}} \exp \left(-\frac{\varepsilon \eta^{2}}{4 t}\right) d \eta \leq 2\left(4 \pi \varepsilon^{-1} t\right)^{-\frac{1}{2}} L \leq C\left(\varepsilon^{-1} t\right)^{-\frac{1}{2}}
\end{aligned}
$$

for $x \in \mathbb{R}^{N-1} \times(0, L), t>0$ and $\varepsilon>0$. For any $\phi \in L^{\infty}\left(\mathbb{R}_{+}^{N}\right)$, by (1.4) and (2.6) we have

$$
\left|\left[S_{1}\left(\varepsilon^{-1} t\right) \phi\right](x)\right| \leq \int_{\mathbb{R}_{+}^{N}} \Gamma_{D}\left(x, y, \varepsilon^{-1} t\right)|\phi(y)| d y \leq C\left(\varepsilon^{-1} t\right)^{-\frac{1}{2}}\|\phi\|_{L^{\infty}}
$$

for $x \in \mathbb{R}^{N-1} \times(0, L), t>0$ and $\varepsilon>0$. This implies (2.3), and the proof of Lemma 2.1 is complete.

Next we recall some properties of $S_{2}(t) \psi$.

$\left(\mathrm{P}_{1}\right)$ Let $\psi \in L^{r}\left(\mathbb{R}^{N-1}\right)$ for some $r \in[1, \infty]$ and $t, t^{\prime}>0$. Then

$$
\begin{aligned}
& {\left[S_{2}(t) \psi\right]\left(x^{\prime}, x_{N}\right)=\left[S_{2}\left(t+x_{N}\right) \psi\right]\left(x^{\prime}, 0\right),} \\
& {\left[S_{2}\left(t+t^{\prime}\right) \psi\right](x)=\left[S_{2}(t)\left(S_{2}\left(t^{\prime}\right) \psi\right)\right](x),}
\end{aligned}
$$

for $x=\left(x^{\prime}, x_{N}\right) \in \overline{\mathbb{R}_{+}^{N}}$. Furthermore,

$$
\lim _{t \rightarrow 0}\left|S_{2}(t) \psi-\psi\right|_{r}=0 \quad \text { if } 1 \leq r<\infty
$$

$\left(\mathrm{P}_{2}\right)$ For any $1 \leq q \leq r \leq \infty$,

$$
\left|S_{2}(t) \psi\right|_{L^{r}} \leq C t^{-(N-1)\left(\frac{1}{q}-\frac{1}{r}\right)}|\psi|_{L^{q}}, \quad t>0,
$$

for all $\psi \in L^{q}\left(\mathbb{R}^{N-1}\right)$. In particular, if $q=r$, then

$$
\sup _{t>0}\left|S_{2}(t) \psi\right|_{L^{r}} \leq|\psi|_{L^{r}}
$$

Properties $\left(\mathrm{P}_{1}\right)$ and $\left(\mathrm{P}_{2}\right)$ easily follow from (1.5) (see e.g. [15]) and imply that

$$
\sup _{t>0}\left\|S_{2}(t) \psi\right\|_{L^{\infty}} \leq|\psi|_{L^{\infty}}
$$

for all $\psi \in L^{\infty}\left(\mathbb{R}^{N-1}\right)$. Furthermore, by a similar argument as in the proof of property $\left(G_{2}\right)$ we have:

$\left(\mathrm{P}_{3}\right)$ Let $\psi \in L^{q}\left(\mathbb{R}^{N-1}\right)$ with $1 \leq q \leq \infty$. Then, for any $T>0, S_{2}(t) \psi$ is bounded and smooth in $\overline{\mathbb{R}_{+}^{N}} \times(T, \infty)$. 
Lemma 2.2 Let $\psi \in L^{\infty}\left(\mathbb{R}^{N-1}\right)$. Set

$$
D_{\varepsilon}[\psi](x, t):=\int_{0}^{t}\left[S_{1}\left(\varepsilon^{-1}(t-s)\right) F_{1}[\psi](s)\right](x) d s
$$

for $x \in \overline{\mathbb{R}_{+}^{N}}, t>0$ and $\varepsilon>0$. Then $D_{\varepsilon}[\psi]$ and $\partial_{x_{N}} D_{\varepsilon}[\psi]$ are bounded and smooth in $\overline{\mathbb{R}_{+}^{N}} \times(T, \infty)$ for any $T>0$. Furthermore, there exists $C>0$ such that

$$
\left\|D_{\varepsilon}[\psi](t)\right\|_{L^{\infty}} \leq C t^{\frac{1}{4}}\left(\varepsilon^{\frac{1}{2}}+t^{\frac{3}{4}}\right)|\psi|_{L^{\infty}}
$$

for $t>0$ and $\varepsilon>0$. Moreover,

$$
\lim _{\varepsilon \rightarrow 0} \sup _{t \in\left(0, T_{1}\right)}\left\|D_{\varepsilon}[\psi](t)\right\|_{L^{\infty}\left(\mathbb{R}^{N-1} \times(0, L)\right)}=0
$$

for $T_{1}>0$ and $L>0$.

Proof. We prove (2.10) first. Since

$$
\partial_{t} P\left(x^{\prime}, x_{N}, t\right)=\frac{1}{x_{N}+t} \frac{\left|x^{\prime}\right|^{2}-(N-1)\left(x_{N}+t\right)^{2}}{\left|x^{\prime}\right|^{2}+\left(x_{N}+t\right)^{2}} P\left(x^{\prime}, x_{N}, t\right),
$$

it follows that

$$
\left|\partial_{t} P\left(x^{\prime}, x_{N}, t\right)\right| \leq C\left(x_{N}+t\right)^{-1} P\left(x^{\prime}, x_{N}, t\right) .
$$

By (1.12), (2.7) and (2.12) we have

$$
\begin{aligned}
\left\|F_{1}[\psi]\left(\cdot, y_{N}, s\right)\right\|_{L^{\infty}\left(\mathbb{R}^{N-1}\right)} & \leq C\left(y_{N}+s\right)^{-1}\left\|S_{2}\left(s+y_{N}\right) \psi\right\|_{L^{\infty}\left(\mathbb{R}^{N-1}\right)} \\
& \leq C\left(y_{N}+s\right)^{-1}|\psi|_{L^{\infty}}
\end{aligned}
$$

for $y_{N} \in[0, \infty)$ and $s>0$. Since

$$
\left(y_{N}+s\right)^{-1} \leq \begin{cases}y_{N}^{-\frac{3}{4}} s^{-\frac{1}{4}} & \text { for } 0 \leq y_{N} \leq 1, \\ 1 & \text { for } y_{N}>1\end{cases}
$$

by (1.3), (2.9) and (2.13) we see that

$$
\begin{aligned}
& \left|D_{\varepsilon}[\psi](x, t)\right| \leq \int_{0}^{t} \int_{\mathbb{R}_{+}^{N}} \Gamma_{D}\left(x, y, \tau_{\varepsilon}\right)\left|F_{1}[\psi](y, s)\right| d y d s \\
& \leq C \int_{0}^{t} \int_{0}^{\infty} \Gamma_{1}\left(x_{N}-y_{N}, \tau_{\varepsilon}\right)\left\|F_{1}[\psi]\left(\cdot, y_{N}, s\right)\right\|_{L^{\infty}\left(\mathbb{R}^{N-1}\right)} d y_{N} d s \\
& \leq C|\psi|_{L^{\infty}} \int_{0}^{t} \int_{0}^{\infty} \tau_{\varepsilon}^{-\frac{1}{2}} \exp \left(-\frac{\left(x_{N}-y_{N}\right)^{2}}{4 \tau_{\varepsilon}}\right)\left(y_{N}+s\right)^{-1} d y_{N} d s \\
& \leq C|\psi|_{L^{\infty}}\left\{\int_{0}^{t} \tau_{\varepsilon}^{-\frac{1}{2}} s^{-\frac{1}{4}} \int_{0}^{1} y_{N}^{-\frac{3}{4}} d y_{N} d s+\int_{0}^{t} d s\right\} \\
& \leq C|\psi|_{L^{\infty}}\left\{\varepsilon^{\frac{1}{2}} \int_{0}^{t}(t-s)^{-\frac{1}{2}} s^{-\frac{1}{4}} d s+t\right\} \\
& \leq C|\psi|_{L^{\infty}}\left(\varepsilon^{\frac{1}{2}} t^{\frac{1}{4}}+t\right)=C|\psi|_{L^{\infty}} t^{\frac{1}{4}}\left(\varepsilon^{\frac{1}{2}}+t^{\frac{3}{4}}\right)
\end{aligned}
$$


for $x \in \overline{\mathbb{R}_{+}^{N}}, t>0$ and $\varepsilon>0$, where $\tau_{\varepsilon}:=\varepsilon^{-1}(t-s)$. Here $\Gamma_{1}$ is the one-dimensional Gauss kernel. This implies (2.10).

We prove (2.11). Let $L>0$. Similarly to (2.15), we obtain

$$
\begin{aligned}
& \quad\left|D_{\varepsilon}[\psi](x, t)\right| \leq \int_{0}^{t} \int_{\mathbb{R}_{+}^{N}} \Gamma_{D}\left(x, y, \tau_{\varepsilon}\right)\left|F_{1}[\psi](y, s)\right| d y d s \\
& \leq C \int_{0}^{t} \int_{0}^{\infty}\left(\Gamma_{1}\left(x_{N}-y_{N}, \tau_{\varepsilon}\right)-\Gamma_{1}\left(x_{N}+y_{N}, \varepsilon^{-1}(t-s)\right)\right) \\
& \times\left\|F_{1}[\psi]\left(\cdot, y_{N}, s\right)\right\|_{L^{\infty}\left(\mathbb{R}^{N-1}\right)} d y_{N} d s \\
& \leq C|\psi|_{L^{\infty}} \int_{0}^{t} \tau_{\varepsilon}^{-\frac{1}{2}} \int_{0}^{\infty}\left(y_{N}+s\right)^{-1} \\
& \quad \times\left[\exp \left(-\frac{\left(x_{N}-y_{N}\right)^{2}}{4 \tau_{\varepsilon}}\right)-\exp \left(-\frac{\left(x_{N}+y_{N}\right)^{2}}{4 \tau_{\varepsilon}}\right)\right] d y_{N} d s
\end{aligned}
$$

for $x \in \overline{\mathbb{R}_{+}^{N}}, t>0$ and $\varepsilon>0$. This together with (2.6), (2.13) and (2.14) implies that

$$
\begin{aligned}
& \left|D_{\varepsilon}[\psi](x, t)\right| \\
& \leq C|\psi|_{L^{\infty}}\left\{\int_{0}^{t} \tau_{\varepsilon}^{-\frac{1}{2}} s^{-\frac{1}{4}} \int_{0}^{1} y_{N}^{-\frac{3}{4}} d y_{N} d s\right. \\
& \left.+\int_{0}^{t} \tau_{\varepsilon}^{-\frac{1}{2}} \int_{1}^{\infty}\left[\exp \left(-\frac{\left(x_{N}-y_{N}\right)^{2}}{4 \tau_{\varepsilon}}\right)-\exp \left(-\frac{\left(x_{N}+y_{N}\right)^{2}}{4 \tau_{\varepsilon}}\right)\right] d y_{N} d s\right\} \\
& \leq C|\psi|_{L^{\infty}}\left\{\int_{0}^{t} \tau_{\varepsilon}^{-\frac{1}{2}} s^{-\frac{1}{4}} d s+\int_{0}^{t} \tau_{\varepsilon}^{-\frac{1}{2}} d s\right\} \\
& \leq C|\psi|_{L^{\infty}} \varepsilon^{\frac{1}{2}}\left\{\int_{0}^{t}(t-s)^{-\frac{1}{2}} s^{-\frac{1}{4}} d s+\int_{0}^{t}(t-s)^{-\frac{1}{2}} d s\right\} \\
& \leq C|\psi|_{L^{\infty}}\left(\varepsilon^{\frac{1}{2}} t^{\frac{1}{4}}+(\varepsilon t)^{\frac{1}{2}}\right)
\end{aligned}
$$

for $x \in \mathbb{R}^{N-1} \times(0, L), t>0$ and $\varepsilon>0$. Thus (2.11) holds.

On the other hand, it follows from the semigroup property of $S_{1}(t)$ that

$$
\begin{aligned}
& D_{\varepsilon}[\psi](x, t)=\int_{0}^{t}\left[S_{1}\left(\varepsilon^{-1}(t-s)\right) F_{1}[\psi](s)\right](x) d s \\
& =S_{1}\left(\varepsilon^{-1}(t-T / 2)\right) D_{\varepsilon}[\psi](x, T / 2)+\int_{T / 2}^{t}\left[S_{1}\left(\varepsilon^{-1}(t-s)\right) F_{1}[\psi](s)\right](x) d s
\end{aligned}
$$

for $x \in \overline{\mathbb{R}_{+}^{N}}$ and $0<T<t<\infty$. Then, by (2.9) and $\left(\mathrm{G}_{2}\right)$ we see that

$$
S_{1}\left(\varepsilon^{-1}(t-T / 2)\right) D_{\varepsilon}[\psi](x, T / 2)
$$

is bounded and smooth in $\overline{\mathbb{R}_{+}^{N}} \times(T, \infty)$. Furthermore, by (2.13) we apply the same argument as in [23, Section 3, Chapter 1] to see that

$$
\int_{T / 2}^{t}\left[S_{1}\left(\varepsilon^{-1}(t-s)\right) F_{1}[\psi](s)\right](x) d s
$$


is also bounded and smooth in $\overline{\mathbb{R}_{+}^{N}} \times(T, \infty)$. (See also [14, Proposition 5.2] and [28, Lemma 2.1].) Therefore we deduce that $D_{\varepsilon}[\psi]$ and $\partial_{x_{N}} D_{\varepsilon}[\psi]$ are bounded and smooth in $\overline{\mathbb{R}_{+}^{N}} \times(T, \infty)$. Thus Lemma 2.2 follows.

Lemma 2.3 Let $0 \leq \alpha<1$. Then there exists $C>0$ such that

$$
\int_{0}^{\infty} \frac{|x \pm y|}{t} \Gamma_{1}(x \pm y, t) y^{-\alpha} d y \leq C t^{-\frac{\alpha+1}{2}}
$$

for $x \geq 0$ and $t>0$. Here $\Gamma_{1}$ is the one-dimensional Gauss kernel.

Proof. Let $x \geq 0$ and $t>0$. It follows that

$$
\begin{aligned}
& \int_{0}^{\infty} \frac{|x-y|}{t} \Gamma_{1}(x-y, t) y^{-\alpha} d y \\
& =(4 \pi t)^{-\frac{1}{2}}\left(\int_{0}^{x_{2}}+\int_{x / 2}^{\infty}\right) \frac{|x-y|}{t} \exp \left(-\frac{|x-y|^{2}}{4 t}\right) y^{-\alpha} d y .
\end{aligned}
$$

Since $y^{-1} \leq|x-y|^{-1}$ for $0 \leq x \leq 2 y$, we have

$$
\begin{aligned}
& \int_{0}^{\infty} \frac{|x-y|}{t} \Gamma_{1}(x-y, t) y^{-\alpha} d y \\
& \leq C t^{-\frac{1}{2}} \frac{x}{t} \exp \left(-\frac{x^{2}}{16 t}\right) \int_{0}^{x / 2} y^{-\alpha} d y_{N} \\
& \quad+C t^{-\frac{3}{2}+\frac{1-\alpha}{2}} \int_{x / 2}^{\infty}\left(\frac{|x-y|}{t^{1 / 2}}\right)^{1-\alpha} \exp \left(-\frac{|x-y|^{2}}{4 t}\right) d y \\
& \leq C t^{-\frac{3}{2}} x^{2-\alpha} \exp \left(-\frac{x^{2}}{16 t}\right)+C t^{-1+\frac{1-\alpha}{2}} \leq C t^{-\frac{\alpha+1}{2}}
\end{aligned}
$$

Since $y^{-1} \leq 2(x+y)^{-1}$ for $0 \leq x \leq y$, similarly to (2.18), we obtain

$$
\int_{0}^{\infty} \frac{x+y}{t} \Gamma_{1}(x+y, t) y^{-\alpha} d y \leq C t^{-\frac{\alpha+1}{2}} .
$$

Thus (2.17) holds and Lemma 2.3 follows.

Lemma 2.4 Let $\psi \in L^{\infty}\left(\mathbb{R}^{N-1}\right)$. Then there exists $C>0$ such that

$$
\left\|\partial_{x_{N}} D_{\varepsilon}[\psi](t)\right\|_{L^{\infty}} \leq C \varepsilon^{\frac{3}{4}} t^{-\frac{1}{4}}|\psi|_{L^{\infty}}
$$

for $t>0$ and $\varepsilon>0$.

Proof. By (2.4), (2.9) and (2.13) we see that

$$
\begin{aligned}
& \left|\partial_{x_{N}} D_{\varepsilon}[\psi](x, t)\right| \\
& \leq \int_{0}^{t} \int_{\mathbb{R}_{+}^{N}}\left|K\left(x, y, \tau_{\varepsilon}\right)\right|\left|F_{1}[\psi](y, s)\right| d y d s \\
& \leq C \int_{0}^{t} \int_{0}^{\infty} \tilde{K}\left(x_{N}, y_{N}, \tau_{\varepsilon}\right)\left\|F_{1}[\psi]\left(\cdot, y_{N}, s\right)\right\|_{L^{\infty}\left(\mathbb{R}^{N-1}\right)} d y_{N} d s \\
& \leq C|\psi|_{L^{\infty}} \int_{0}^{t} s^{-\frac{1}{2}} \int_{0}^{\infty} \tilde{K}\left(x_{N}, y_{N}, \tau_{\varepsilon}\right) y_{N}^{-\frac{1}{2}} d y_{N} d s
\end{aligned}
$$


for $x \in \overline{\mathbb{R}_{+}^{N}}, t>0$ and $\varepsilon>0$, where $\tau_{\varepsilon}:=\varepsilon^{-1}(t-s)$ and

$$
\tilde{K}\left(x_{N}, y_{N}, t\right)=\frac{\left|x_{N}-y_{N}\right|}{t} \Gamma_{1}\left(x_{N}-y_{N}, t\right)+\frac{x_{N}+y_{N}}{t} \Gamma_{1}\left(x_{N}+y_{N}, t\right)
$$

for $x_{N} \geq 0, y_{N}>0$ and $t>0$. By (2.17) with $\alpha=1 / 2$ and (2.20) we deduce that

$$
\begin{aligned}
\left|\partial_{x_{N}} D_{\varepsilon}[\psi](x, t)\right| & \leq C|\psi|_{L^{\infty}} \int_{0}^{t} s^{-\frac{1}{2}} \tau_{\varepsilon}^{-\frac{3}{4}} d s \\
& =C|\psi|_{L^{\infty}} \int_{0}^{t} s^{-\frac{1}{2}}\left(\varepsilon^{-1}(t-s)\right)^{-\frac{3}{4}} d s \leq C \varepsilon^{\frac{3}{4}} t^{-\frac{1}{4}}|\psi|_{L^{\infty}}
\end{aligned}
$$

for $x \in \overline{\mathbb{R}_{+}^{N}}, t>0$ and $\varepsilon>0$. Thus (2.19) follows.

\section{Proof of Theorem 1.1}

We introduce some notation. Let $T>0$ and $\varepsilon \in(0,1)$. Set

$$
X_{T}:=\left\{v, \partial_{x_{N}} v \in C\left(\overline{\mathbb{R}_{+}^{N}} \times(0, T)\right):\|v\|_{X_{T}}<\infty\right\}, \quad\|v\|_{X_{T}}:=\sup _{0<t<T} E_{\varepsilon}[v](t),
$$

where

$$
E_{\varepsilon}[v](t):=\|v(t)\|_{L^{\infty}}+\left(\varepsilon^{-1} t\right)^{\frac{1}{2}}\left\|\partial_{x_{N}} v(t)\right\|_{L^{\infty}} .
$$

Then $X_{T}$ is a Banach space equipped with the norm $\|\cdot\|_{X_{T}}$. For the proof of Theorem 1.1 we apply the Banach contraction mapping principle in $X_{T}$ to find a fixed point of

$$
Q_{\varepsilon}[v](t):=S_{1}\left(\varepsilon^{-1} t\right) \Phi-D_{\varepsilon}\left[\varphi_{b}\right](t)-\int_{0}^{t} S_{1}\left(\varepsilon^{-1}(t-s)\right) F_{2}[v](s) d s,
$$

where $\Phi, F_{2}[v]$ and $D_{\varepsilon}\left[\varphi_{b}\right]$ are as in (1.9), (1.13) and (2.9), respectively.

Lemma 3.1 There exists $C>0$ such that

$$
F_{2}[v](x, t) \leq C \varepsilon^{\frac{1}{2}}\left(t^{-\frac{1}{2}}+h\left(x_{N}, t\right)\right)\|v\|_{X_{T}}
$$

for $x \in \mathbb{R}_{+}^{N}, 0<t<T, \varepsilon \in(0,1)$ and $v \in X_{T}$. Here

$$
h\left(x_{N}, t\right):=x_{N}^{-\frac{3}{4}} t^{\frac{1}{4}} \quad \text { if } \quad 0<x_{N} \leq 1 \quad \text { and } \quad h\left(x_{N}, t\right):=x_{N}^{-\frac{1}{2}} \quad \text { if } \quad x_{N}>1 .
$$

Proof. Let $T>0, \varepsilon \in(0,1)$ and $v \in X_{T}$. It follows from (1.13) that

$$
F_{2}[v](x, t)=F_{2}^{\prime}[v](x, t)+F_{2}^{\prime \prime}[v](x, t)
$$

for $x \in \mathbb{R}_{+}^{N}$ and $t>0$, where

$$
\begin{aligned}
& F_{2}^{\prime}[v](x, t):=\int_{\mathbb{R}^{N-1}} P\left(x^{\prime}-y^{\prime}, x_{N}, 0\right) \partial_{x_{N}} v\left(y^{\prime}, 0, t\right) d y^{\prime}, \\
& F_{2}^{\prime \prime}[v](x, t):=\int_{0}^{t} \int_{\mathbb{R}^{N-1}} \partial_{t} P\left(x^{\prime}-y^{\prime}, x_{N}, t-s\right) \partial_{x_{N}} v\left(y^{\prime}, 0, s\right) d y^{\prime} d s .
\end{aligned}
$$


Since $v \in X_{T}$, by (1.5) and (2.7) we see that

$$
\begin{aligned}
\left|F_{2}^{\prime}[v](x, t)\right| & \leq\left\|S_{2}\left(x_{N}\right) \partial_{x_{N}} v(\cdot, 0, t)\right\|_{L^{\infty}\left(\mathbb{R}^{N-1}\right)} \\
& \leq\left|\partial_{x_{N}} v(t)\right|_{L^{\infty}} \leq \varepsilon^{\frac{1}{2}} t^{-\frac{1}{2}}\|v\|_{X_{T}}
\end{aligned}
$$

for $x \in \mathbb{R}_{+}^{N}$ and $t>0$. On the other hand, it follows from (2.12) that

$$
\left|\partial_{t} P\left(x^{\prime}, x_{N}, t\right)\right| \leq \begin{cases}C P\left(x^{\prime}, x_{N}, t\right) x_{N}^{-\frac{3}{4}} t^{-\frac{1}{4}} & \text { if } \quad x_{N} \leq 1 \\ C P\left(x^{\prime}, x_{N}, t\right) x_{N}^{-\frac{1}{2}} t^{-\frac{1}{2}} & \text { if } \quad x_{N}>1\end{cases}
$$

for $x \in \mathbb{R}_{+}^{N}$ and $t>0$. Then we obtain

$$
\begin{aligned}
& \left|F_{2}^{\prime \prime}[v](x, t)\right| \\
& \leq C x_{N}^{-\frac{3}{4}} \int_{0}^{t}(t-s)^{-\frac{1}{4}} \int_{\mathbb{R}^{N-1}} P\left(x^{\prime}-y^{\prime}, x_{N}, t-s\right)\left|\partial_{x_{N}} v\left(y^{\prime}, 0, s\right)\right| d y^{\prime} d s \\
& \leq C \varepsilon^{\frac{1}{2}} x_{N}^{-\frac{3}{4}}\left(\sup _{0<s<t}\left(\varepsilon^{-1} s\right)^{\frac{1}{2}}\left|\partial_{x_{N}} v(s)\right|_{L^{\infty}}\right) \int_{0}^{t}(t-s)^{-\frac{1}{4}} s^{-\frac{1}{2}} d s \\
& \leq C \varepsilon^{\frac{1}{2}} x_{N}^{-\frac{3}{4}} t^{\frac{1}{4}}\|v\|_{X_{T}}=C \varepsilon^{\frac{1}{2}} h\left(x_{N}, t\right)\|v\|_{X_{T}}
\end{aligned}
$$

for $x^{\prime} \in \mathbb{R}^{N-1}, 0<x_{N} \leq 1$ and $0<t<T$. Similarly, we deduce that

$$
\begin{aligned}
& \left|F_{2}^{\prime \prime}[v](x, t)\right| \\
& \leq C \int_{0}^{t}(t-s)^{-\frac{1}{2}} \int_{\mathbb{R}_{+}^{N}} P\left(x^{\prime}-y^{\prime}, x_{N}, t-s\right)\left|\partial_{x_{N}} v\left(y^{\prime}, 0, s\right)\right| d y^{\prime} d s \\
& \leq C \varepsilon^{\frac{1}{2}} x_{N}^{-\frac{1}{2}}\left(\sup _{0<s<t}\left(\varepsilon^{-1} s\right)^{\frac{1}{2}}\left|\partial_{x_{N}} v(s)\right|_{L^{\infty}}\right) \int_{0}^{t}(t-s)^{-\frac{1}{2}} s^{-\frac{1}{2}} d s \\
& \leq C \varepsilon^{\frac{1}{2}} x_{N}^{-\frac{1}{2}}\|v\|_{X_{T}}=C \varepsilon^{\frac{1}{2}} h\left(x_{N}, t\right)\|v\|_{X_{T}}
\end{aligned}
$$

for $x^{\prime} \in \mathbb{R}^{N-1}, x_{N}>1$ and $0<t<T$. Therefore, by (3.3), (3.4), (3.5) and (3.6) we obtain (3.2). Thus Lemma 3.1 follows.

Lemma 3.2 For any $v \in X_{T}$ and $\varepsilon \in(0,1)$, set

$$
\tilde{D}_{\varepsilon}[v](t):=\int_{0}^{t} S_{1}\left(\varepsilon^{-1}(t-s)\right) F_{2}[v](s) d s .
$$

Then there exists $T_{*}=T_{*}(N)>0$ such that

$$
\left\|\tilde{D}_{\varepsilon}[v]\right\|_{X_{T_{*}}} \leq \frac{1}{4}\|v\|_{X_{T_{*}}}
$$

for $v \in X_{T_{*}}$ and $\varepsilon \in(0,1)$. Furthermore, $\tilde{D}_{\varepsilon}[v]$ and $\partial_{x_{N}} \tilde{D}_{\varepsilon}[v]$ are bounded and smooth in $\overline{\mathbb{R}_{+}^{N}} \times\left(\tau, T_{*}\right)$ for any $0<\tau<T_{*}$. 
Proof. Let $T>0$. By (2.9) and (3.7) we see that $\tilde{D}_{\varepsilon}$ is defined analogously as $D_{\varepsilon}$ with $F_{1}$ replaced by $F_{2}$. Then it follows from (2.15) and (3.2) that

$$
\begin{aligned}
& \left|\tilde{D}_{\varepsilon}[v](x, t)\right| \\
& \leq C \int_{0}^{t} \int_{0}^{\infty} \Gamma_{1}\left(x_{N}-y_{N}, \tau_{\varepsilon}\right)\left\|F_{2}[v]\left(\cdot, y_{N}, s\right)\right\|_{L^{\infty}\left(\mathbb{R}^{N-1}\right)} d y_{N} d s \\
& \leq C \varepsilon^{\frac{1}{2}}\|v\|_{X_{T}} \int_{0}^{t} \int_{0}^{\infty} \tau_{\varepsilon}^{-\frac{1}{2}} \exp \left(-\frac{\left(x_{N}-y_{N}\right)^{2}}{4 \tau_{\varepsilon}}\right)\left(s^{-\frac{1}{2}}+h\left(y_{N}, s\right)\right) d y_{N} d s \\
& \leq C \varepsilon^{\frac{1}{2}}\|v\|_{X_{T}}\left\{\int_{0}^{t} s^{-\frac{1}{2}} d s+\int_{0}^{t} \tau_{\varepsilon}^{-\frac{1}{2}} s^{\frac{1}{4}} \int_{0}^{1} y_{N}^{-\frac{3}{4}} d y_{N} d s\right. \\
& \left.\quad+\int_{0}^{t} \int_{1}^{\infty} \tau_{\varepsilon}^{-\frac{1}{2}} \exp \left(-\frac{\left(x_{N}-y_{N}\right)^{2}}{4 \tau_{\varepsilon}}\right) d y_{N} d s\right\} \\
& \leq C \varepsilon^{\frac{1}{2}}\|v\|_{X_{T}}\left\{t^{\frac{1}{2}}+\varepsilon^{\frac{1}{2}} \int_{0}^{t}(t-s)^{-\frac{1}{2}} s^{\frac{1}{4}} d s+t\right\} \leq C \varepsilon^{\frac{1}{2}}\|v\|_{X_{T}}\left(t^{\frac{1}{2}}+\varepsilon^{\frac{1}{2}} t^{\frac{3}{4}}+t\right)
\end{aligned}
$$

for $x \in \overline{\mathbb{R}_{+}^{N}}$ and $0<t<T$, where $\tau_{\varepsilon}=\varepsilon^{-1}(t-s)$. Then, taking a sufficiently small $T>0$ if necessary, we obtain

$$
\sup _{0<t<T}\left\|\tilde{D}_{\varepsilon}[v](t)\right\|_{L^{\infty}} \leq \frac{1}{8}\|v\|_{X_{T}} .
$$

On the other hand, similarly to (2.20), by (3.2) we see that

$$
\begin{aligned}
& \left|\partial_{x_{N}} \tilde{D}_{\varepsilon}[v](x, t)\right| \\
& \leq C \int_{0}^{t} \int_{0}^{\infty} \tilde{K}\left(x_{N}, y_{N}, \tau_{\varepsilon}\right)\left\|F_{2}[v]\left(\cdot, y_{N}, s\right)\right\|_{L^{\infty}\left(\mathbb{R}^{N-1}\right)} d y_{N} d s \\
& \leq C \varepsilon^{\frac{1}{2}}\|v\|_{X_{T}} \int_{0}^{t} \int_{0}^{\infty} \tilde{K}\left(x_{N}, y_{N}, \tau_{\varepsilon}\right)\left(s^{-\frac{1}{2}}+h\left(y_{N}, s\right)\right) d y_{N} d s \\
& \leq C \varepsilon^{\frac{1}{2}}\|v\|_{X_{T}} \int_{0}^{t} \int_{0}^{\infty} \tilde{K}\left(x_{N}, y_{N}, \tau_{\varepsilon}\right)\left(s^{-\frac{1}{2}}+y_{N}^{-\frac{3}{4}} s^{\frac{1}{4}}+y_{N}^{-\frac{1}{2}}\right) d y_{N} d s
\end{aligned}
$$

for $x \in \overline{\mathbb{R}_{+}^{N}}$ and $0<t<T$, where $\tilde{K}$ is the function given by (2.21). By (2.17) we have

$$
\begin{aligned}
& \left|\partial_{x_{N}} \tilde{D}_{\varepsilon}[v](x, t)\right| \leq C \varepsilon^{\frac{1}{2}}\|v\|_{X_{T}}\left(\int_{0}^{t} s^{-\frac{1}{2}} \tau_{\varepsilon}^{-\frac{1}{2}} d s+\int_{0}^{t} s^{\frac{1}{4}} \tau_{\varepsilon}^{-\frac{7}{8}} d s+\int_{0}^{t} \tau_{\varepsilon}^{-\frac{3}{4}} d s\right) \\
& =C \varepsilon^{\frac{1}{2}}\|v\|_{X_{T}}\left(\int_{0}^{t} s^{-\frac{1}{2}}\left(\varepsilon^{-1}(t-s)\right)^{-\frac{1}{2}} d s\right. \\
& \left.+\int_{0}^{t} s^{\frac{1}{4}}\left(\varepsilon^{-1}(t-s)\right)^{-\frac{7}{8}} d s+\int_{0}^{t}\left(\varepsilon^{-1}(t-s)\right)^{-\frac{3}{4}} d s\right) \\
& \leq C \varepsilon^{\frac{1}{2}}\|v\|_{X_{T}}\left(\varepsilon^{\frac{1}{2}}+\varepsilon^{\frac{7}{8}} t^{\frac{3}{8}}+\varepsilon^{\frac{3}{4}} t^{\frac{1}{4}}\right) \\
& \leq C\left(\varepsilon^{-1} t\right)^{-\frac{1}{2}}\|v\|_{X_{T}}\left((\varepsilon t)^{\frac{1}{2}}+(\varepsilon t)^{\frac{7}{8}}+(\varepsilon t)^{\frac{3}{4}}\right)
\end{aligned}
$$


for $x \in \overline{\mathbb{R}_{+}^{N}}$ and $0<t<T$. Taking a sufficiently small $T>0$ if necessary, we see that

$$
\sup _{0<t<T}\left(\varepsilon^{-1} t\right)^{\frac{1}{2}}\left\|\partial_{x_{N}} \tilde{D}_{\varepsilon}[v](t)\right\|_{L^{\infty}} \leq \frac{1}{8}\|v\|_{X_{T}} .
$$

Therefore, by (3.9) and (3.10) we have (3.8). Furthermore, by (3.2) we apply a similar argument as in the proof of Lemma 2.2 and deduce that $\tilde{D}_{\varepsilon}[v]$ and $\partial_{x_{N}} \tilde{D}_{\varepsilon}[v]$ are bounded and smooth in $\overline{\mathbb{R}_{+}^{N}} \times(\tau, T)$ for any $0<\tau<T$. Thus Lemma 3.2 follows.

Now we are ready to complete the proof of Theorem 1.1,

Proof of Theorem 1.1. Let

$$
m:=16 \max \left\{\|\varphi\|_{L^{\infty}},\left|\varphi_{b}\right|_{L^{\infty}}\right\} .
$$

Let $T_{*}>0$ be as in Lemma 3.2 and $v \in X_{T_{*}}$ with $\|v\|_{X_{T_{*}}} \leq m$. Then, by property $\left(G_{2}\right)$, Lemmata 2.2 and 3.2 we see that $Q_{\varepsilon}[v] \in X_{T_{*}}$. Since it follows from (1.9) and (2.8) that

$$
\|\Phi\|_{L^{\infty}} \leq\|\varphi\|_{L^{\infty}}+\left|\varphi_{b}\right|_{L^{\infty}}
$$

by (2.1), (2.2) and (3.11) we have

$$
\left\|S_{1}\left(\varepsilon^{-1} t\right) \Phi\right\|_{L^{\infty}}+\left(\varepsilon^{-1} t\right)^{\frac{1}{2}}\left\|\partial_{x_{N}}\left[S_{1}\left(\varepsilon^{-1} t\right) \Phi\right]\right\|_{L^{\infty}} \leq 2\|\Phi\|_{L^{\infty}} \leq \frac{m}{4}
$$

for $0<t<T_{*}$. Furthermore, by (2.10) and (2.19), taking a sufficiently small $T_{*}>0$ if necessary, we see that

$$
\left\|D_{\varepsilon}\left[\varphi_{b}\right](t)\right\|_{L^{\infty}}+\left(\varepsilon^{-1} t\right)^{\frac{1}{2}}\left\|\partial_{x_{N}} D_{\varepsilon}\left[\varphi_{b}\right](t)\right\|_{L^{\infty}} \leq\left.\left. C T_{*}^{\frac{1}{4}}\left(1+T_{*}^{\frac{3}{4}}\right)\right|_{b}\right|_{\infty} \leq \frac{m}{4}
$$

for $0<t<T_{*}$. Lemma 3.2 together with (3.1), (3.12) and (3.13) implies that

$$
\left\|Q_{\varepsilon}[v]\right\|_{X_{T_{*}}} \leq\left\|S_{1}\left(\varepsilon^{-1} \cdot\right) \varphi\right\|_{X_{T_{*}}}+\left\|D_{\varepsilon}\left[\varphi_{b}\right]\right\|_{X_{T_{*}}}+\left\|\tilde{D}_{\varepsilon}[v]\right\|_{X_{T_{*}}} \leq \frac{3 m}{4}<m .
$$

Similarly, we obtain

$$
\left\|Q_{\varepsilon}\left[v_{1}\right]-Q_{\varepsilon}\left[v_{2}\right]\right\|_{X_{T *}}=\left\|\tilde{D}_{\varepsilon}\left[v_{1}\right]-\tilde{D}_{\varepsilon}\left[v_{2}\right]\right\|_{X_{T_{*}}} \leq \frac{1}{4}\left\|v_{1}-v_{2}\right\|_{X_{T_{*}}}
$$

for $v_{i} \in X_{T_{*}}$ with $\left\|v_{i}\right\|_{X_{T_{*}}} \leq m(i=1,2)$. Then, the contraction mapping theorem ensures that there exists a unique $v_{\varepsilon} \in X_{T_{*}}$ with $\left\|v_{\varepsilon}\right\|_{X_{T_{*}}} \leq m$ and

$$
v_{\varepsilon}=Q_{\varepsilon}\left[v_{\varepsilon}\right]=S_{1}\left(\varepsilon^{-1} t\right) \Phi-D_{\varepsilon}\left[\varphi_{b}\right](t)-\tilde{D}_{\varepsilon}\left[v_{\varepsilon}\right](t) \quad \text { in } \quad X_{T_{*}} .
$$

In particular, we see that

$$
\left\|v_{\varepsilon}\right\|_{X_{T_{*}}} \leq C\left(\|\varphi\|_{L^{\infty}}+\left|\varphi_{b}\right|_{L^{\infty}}\right) .
$$

Furthermore, by $\left(G_{2}\right)$ and Lemmata 2.2, 3.2. we see that $v_{\varepsilon}$ is bounded and smooth in $\overline{\mathbb{R}_{+}^{N}} \times\left(T_{1}, T_{*}\right)$ for any $0<T_{1}<T_{*}$. As before, set

$$
w_{\varepsilon}(x, t)=\left[S_{2}(t) \varphi_{b}\right](x)+\int_{0}^{t}\left[S_{2}(t-s) \partial_{x_{N}} v_{\varepsilon}(s)\right](x) d s
$$


for $x \in \overline{\mathbb{R}_{+}^{N}}$ and $t \in\left(0, T_{*}\right)$. By (2.8) and (3.11) we obtain

$$
\begin{aligned}
& \left\|w_{\varepsilon}(t)\right\|_{L^{\infty}} \leq\left\|S_{2}(t) \varphi_{b}\right\|_{L^{\infty}}+\int_{0}^{t}\left\|S_{2}(t-s) \partial_{x_{N}} v_{\varepsilon}(s)\right\|_{L^{\infty}} d s \\
& \quad \leq\left|\varphi_{b}\right|_{L^{\infty}}+\int_{0}^{t}\left|\partial_{x_{N}} v_{\varepsilon}(s)\right|_{L^{\infty}} d s \\
& \quad \leq \frac{m}{16}+\varepsilon^{\frac{1}{2}} m \int_{0}^{t} s^{-\frac{1}{2}} d s \\
& \quad \leq C\left(1+T_{*}^{\frac{1}{2}}\right) m \leq C\left(1+T_{*}^{\frac{1}{2}}\right)\left(\|\varphi\|_{L^{\infty}}+\left|\varphi_{b}\right|_{L^{\infty}}\right)<\infty
\end{aligned}
$$

for all $0<t<T_{*}$. Furthermore, by $\left(\mathrm{P}_{3}\right)$ we apply a similar argument as in Lemma 2.2 and see that $w_{\varepsilon}$ is bounded and smooth in $\overline{\mathbb{R}_{+}^{N}} \times\left(T_{1}, T_{*}\right)$ for any $0<T_{1}<T_{*}$. Therefore we deduce that $\left(v_{\varepsilon}, w_{\varepsilon}\right)$ is a solution of (1.11) in $\mathbb{R}_{+}^{N} \times\left(0, T_{*}\right)$. In addition, by (3.15) and (3.16) we have assertion (a) for any $\tau \in\left(0, T_{*}\right)$. Since $T_{*}$ is independent of $m$, due to the semigroup properties of $S_{1}(t)$ and $S_{2}(t)$, we see that $\left(v_{\varepsilon}, w_{\varepsilon}\right)$ is a global-in-time solution of (1.11) and it satisfies assertion (a) for any $\tau>0$.

Let $\left(\tilde{v}_{\varepsilon}, \tilde{w}_{\varepsilon}\right)$ be a global-in-time solution of (1.11) satisfying (1.14). Since

$$
v_{\varepsilon}-\tilde{v}_{\varepsilon}=Q_{\varepsilon}\left[v_{\varepsilon}\right]-Q_{\varepsilon}\left[\tilde{v}_{\varepsilon}\right]=\tilde{D}_{\varepsilon}\left[v_{\varepsilon}-\tilde{v}_{\varepsilon}\right] \quad \text { in } \quad X_{T_{*}},
$$

by (3.8) we have

$$
\left\|v_{\varepsilon}-\tilde{v}_{\varepsilon}\right\|_{X_{T_{*}}} \leq \frac{1}{4}\left\|v_{\varepsilon}-\tilde{v}_{\varepsilon}\right\|_{X_{T_{*}}} .
$$

This implies that $v_{\varepsilon}=\tilde{v}_{\varepsilon}$ in $X_{T_{*}}$. Repeating this argument, we see that $v_{\varepsilon}=\tilde{v}_{\varepsilon}$ in $X_{T}$ for any $T>0$. Therefore we deduce that $\left(v_{\varepsilon}, w_{\varepsilon}\right)$ is a unique global-in-time solution of (1.11) satisfying (1.14).

It remains to prove assertions (b) and (c). Let $T^{\prime}>0$ and $L>0$. By (1.14) and (2.8) we have

$$
\begin{aligned}
& \left\|w_{\varepsilon}(t)-S_{2}(t) \varphi_{b}\right\|_{L^{\infty}} \leq \int_{0}^{t}\left\|S_{2}(t-s) \partial_{x_{N}} v_{\varepsilon}(s)\right\|_{L^{\infty}} d s \\
& \quad \leq \int_{0}^{t}\left|\partial_{x_{N}} v_{\varepsilon}(s)\right|_{L^{\infty}} d s \leq C\left\|v_{\varepsilon}\right\|_{X_{T^{\prime}}} \varepsilon^{\frac{1}{2}} \int_{0}^{t} s^{-\frac{1}{2}} d s \leq C\left\|v_{\varepsilon}\right\|_{X_{T^{\prime}}} \varepsilon^{\frac{1}{2}} T^{\prime \frac{1}{2}}
\end{aligned}
$$

for all $t \in\left(0, T^{\prime}\right)$. This implies assertion (c). On the other hand, since $\tilde{D}_{\varepsilon}\left[v_{\varepsilon}\right]$ is given with 
$F_{1}[\psi]$ replaced by $F_{2}\left[v_{\varepsilon}\right]$, by $(3.2)$ we apply a similar argument as in (2.16) to obtain

$$
\begin{aligned}
& \left|\tilde{D}_{\varepsilon}\left[v_{\varepsilon}\right](x, t)\right| \leq C \int_{0}^{t} \int_{0}^{\infty}\left(\Gamma_{1}\left(x_{N}-y_{N}, \tau_{\varepsilon}\right)-\Gamma_{1}\left(x_{N}+y_{N}, \tau_{\varepsilon}\right)\right) \\
& \times\left\|F_{2}\left[v_{\varepsilon}\right]\left(\cdot, y_{N}, s\right)\right\|_{L^{\infty}\left(\mathbb{R}^{N-1}\right)} d y_{N} d s \\
& \leq C \varepsilon^{\frac{1}{2}}\left\|v_{\varepsilon}\right\|_{X_{T^{\prime}}} \int_{0}^{t} \int_{0}^{\infty}\left(\Gamma_{1}\left(x_{N}-y_{N}, \tau_{\varepsilon}\right)-\Gamma_{1}\left(x_{N}+y_{N}, \tau_{\varepsilon}\right)\right) \\
& \times\left(s^{-\frac{1}{2}}+h\left(y_{N}, s\right)\right) d y_{N} d s \\
& \leq C \varepsilon^{\frac{1}{2}}\left\|v_{\varepsilon}\right\|_{X_{T^{\prime}}} \int_{0}^{t} \int_{0}^{\infty}\left(\Gamma_{1}\left(x_{N}-y_{N}, \tau_{\varepsilon}\right)-\Gamma_{1}\left(x_{N}+y_{N}, \tau_{\varepsilon}\right)\right) \\
& \times\left(s^{-\frac{1}{2}}+\chi_{y_{n} \geq 1}+y_{N}^{-\frac{3}{4}} s^{\frac{1}{4}} \chi_{0 \leq y_{N}<1}\right) d y_{N} d s
\end{aligned}
$$

for $x \in \mathbb{R}^{N-1} \times(0, L), 0<t<T^{\prime}$ and $\varepsilon>0$, where $\tau_{\varepsilon}=\varepsilon^{-1}(t-s)$. By (2.6) we have

$$
\begin{aligned}
& \left|\tilde{D}_{\varepsilon}\left[v_{\varepsilon}\right](x, t)\right| \\
& \leq C \varepsilon^{\frac{1}{2}}\left\|v_{\varepsilon}\right\|_{X_{T^{\prime}}}\left\{\int_{0}^{t}\left(1+s^{-\frac{1}{2}}\right) \tau_{\varepsilon}^{-\frac{1}{2}} d s+\int_{0}^{t} \tau_{\varepsilon}^{-\frac{1}{2}} s^{\frac{1}{4}} \int_{0}^{1} y_{N}^{-\frac{3}{4}} d y_{N} d s\right\} \\
& \leq C \varepsilon^{\frac{1}{2}}\left\|v_{\varepsilon}\right\|_{X_{T^{\prime}}}\left\{\int_{0}^{t}\left(1+s^{-\frac{1}{2}}\right) \tau_{\varepsilon}^{-\frac{1}{2}} d s+\int_{0}^{t} \tau_{\varepsilon}^{-\frac{1}{2}} s^{\frac{1}{4}} d s\right\} \\
& \leq C \varepsilon\left\|v_{\varepsilon}\right\|_{X_{T^{\prime}}}\left\{\int_{0}^{t}\left(1+s^{-\frac{1}{2}}\right)(t-s)^{-\frac{1}{2}} d s+\int_{0}^{t}(t-s)^{-\frac{1}{2}} s^{\frac{1}{4}} d s\right\} \\
& \leq C \varepsilon\left\|v_{\varepsilon}\right\|_{X_{T^{\prime}}}\left(1+t^{\frac{1}{2}}+t^{\frac{3}{4}}\right)
\end{aligned}
$$

for $x \in \mathbb{R}^{N-1} \times(0, L), 0<t<T^{\prime}$ and $\varepsilon>0$. This implies that

$$
\lim _{\varepsilon \rightarrow 0} \sup _{t \in\left(0, T^{\prime}\right)}\left\|\tilde{D}_{\varepsilon}\left[v_{\varepsilon}\right](t)\right\|_{L^{\infty}\left(\mathbb{R}^{N-1} \times(0, L)\right)}=0 .
$$

Therefore, applying (2.3), (2.11) and (3.17) to (3.14), we obtain assertion (b). Thus the proof of Theorem 1.1 is complete.

Proof of Corollary 1.1. Corollary 1.1 immediately follows from Theorem 1.1 and Definition 1.1.

Acknowledgment. The first author was supported in part by the Slovak Research and Development Agency under the contract No. APVV-14-0378 and by the VEGA grant 1/0347/18. Part of this work was carried out while the first author visited the Research Alliance Center for Mathematical Sciences, Tohoku University. The second author was supported in part by the Grant-in-Aid for Scientific Research (A)(No. 15H02058) from Japan Society for the Promotion of Science. The third author was supported by the Grant-in-Aid for Young Scientists (B) (No. 16K17629) from Japan Society for the Promotion of Science. 


\section{References}

[1] E. S. Al-Aidarous, E. O. Alzahrani, H. Ishii and A. M. Younas, Asymptotic analysis for the eikonal equation with the dynamical boundary conditions, Math. Nachr. 287 (2014), $1563-1588$.

[2] J. M. Arrieta, P. Quittner and A. Rodríguez-Bernal, Parabolic problems with nonlinear dynamical boundary conditions and singular initial data, Differ. Integral Equ. 14 (2001), $1487-1510$.

[3] C. Bandle, J. von Below and W. Reichel, Parabolic problems with dynamical boundary conditions: eigenvalue expansions and blow up, Atti Accad. Naz. Lincei Cl. Sci. Fis. Mat. Natur. Rend. Lincei (9) Mat. Appl. 17 (2006), 35-67.

[4] J. von Below and C. D. Coster, A qualitative theory for parabolic problems under dynamical boundary conditions, J. Inequal. Appl. 5 (2000), 467-486.

[5] J. von Below and G. Pincet Mailly, Blow up for reaction diffusion equations under dynamical boundary conditions, Commun. Partial Differ. Equations 28 (2003), 223-247.

[6] J. von Below and G. Pincet Mailly, Blow up for some nonlinear parabolic problems with convection under dynamical boundary conditions, Discrete Contin. Dyn. Syst. 2007, Suppl. (2007), 1031-1041.

[7] J. von Below, G. Pincet Mailly and J.-F. Rault, Growth order and blow up points for the parabolic Burgers equation under dynamical boundary conditions, Discrete Contin. Dyn. Syst., Ser. S 6 (2013), 825-836.

[8] J. Crank, The Mathematics of Diffusion, Clarendon Press, Oxford, second edition (1975).

[9] A. F. M. ter Elst, M. Meyries and J. Rehberg, Parabolic equations with dynamical boundary conditions and source terms on interfaces, Ann. Mat. Pura Appl. 193 (2014), $1295-1318$.

[10] J. Escher, Nonlinear elliptic systems with dynamic boundary conditions, Math. Z. 210 (1992), 413-439.

[11] J. Escher, Quasilinear parabolic systems with dynamical boundary conditions, Commun. Partial Differ. Equ. 18 (1993), 1309-1364.

[12] J. Escher, On the qualitative behaviour of some semilinear parabolic problems, Differ. Integral Equ. 8 (1995), 247-267.

[13] J. Escher, Stable equilibria to elliptic equations in unbounded domains with nonlinear dynamic boundary conditions, Analysis, München 20 (2000), 325-351.

[14] M. Fila, K. Ishige and T. Kawakami, Convergence to the Poisson kernel for the Laplace equation with a nonlinear dynamical boundary condition, Commun. Pure Appl. Anal. 11 (2012), 1285-1301. 
[15] M. Fila, K. Ishige and T. Kawakami, Large-time behavior of solutions of a semilinear elliptic equation with a dynamical boundary condition, Adv. Differential Equations 18 (2013), 69-100.

[16] M. Fila, K. Ishige and T. Kawakami, Existence of positive solutions of a semilinear elliptic equation with a dynamical boundary condition, Calc. Var. PDE 54 (2015), 2059-2078.

[17] M. Fila, K. Ishige and T. Kawakami, Minimal solutions of a semilinear elliptic equation with a dynamical boundary condition, J. Math. Pures Appl. 105 (2016), 788-809.

[18] M. Fila, K. Ishige and T. Kawakami, An exterior nonlinear elliptic problem with a dynamical boundary condition, Rev. Mat. Complutense 30 (2017), 281-312.

[19] M. Fila and P. Poláčik, Global nonexistence without blow-up for an evolution problem, Math. Z. 232 (1999), 531-545.

[20] M. Fila and P. Quittner, Global solutions of the Laplace equation with a nonlinear dynamical boundary condition, Math. Methods Appl. Sci. 20 (1997), 1325-1333.

[21] M. Fila and P. Quittner, Large time behavior of solutions of a semilinear parabolic equation with a nonlinear dynamical boundary condition, Topics in nonlinear analysisThe Herbert Amann anniversary volume, Basel: Birkhäuser, 1999, 251-272.

[22] A. Fiscella and E. Vitillaro, Local Hadamard well-posedness and blow-up for reactiondiffusion equations with non-linear dynamical boundary conditions, Discrete Contin. Dyn. Syst. 33 (2013), 5015-5047.

[23] A. Friedman, Partial Differential Equations of Parabolic Type, Prentice-Hall, Inc., Englewood Cliffs, N. J. 1964.

[24] M. Giga, Y. Giga and J. Saal, Nonlinear Partial Differential Equations, Asymptotic Behavior of Solutions and Self-Similar Solutions, Birkhäuser, Boston, 2010.

[25] Y. Giga and N. Hamamuki, On a dynamic boundary condition for singular degenerate parabolic equations in a half space, Hokkaido University Preprint Series in Mathematics, 1110: 1-38, DOI 10.14943/84298

[26] C. G. Gal and M. Meyries, Nonlinear elliptic problems with dynamical boundary conditions of reactive and reactive-diffusive type, Proc. Lond. Math. Soc. 108 (2014), 13511380 .

[27] T. Hintermann, Evolution equations with dynamic boundary conditions, Proc. R. Soc. Edinburgh Sect. A 113 (1989), 43-60.

[28] K. Ishige, K. Kawakami and K. Kobayashi, Global solutions for a nonlinear integral equation with a generalized heat kernel, Discrete Contin. Dyn. Syst. Ser. S. 7 (2014), $767-783$. 
[29] M. Kirane, Blow-up for some equations with semilinear dynamical boundary conditions of parabolic and hyperbolic type, Hokkaido Math. J. 21 (1992), 221-229.

[30] M. Kirane, E. Nabana and S. I. Pokhozhaev, The absence of solutions of elliptic systems with dynamic boundary conditions, Differ. Equ. 38 (2002), 808-815; translation from Differ. Uravn. 38 (2002), 768-774.

[31] M. Kirane, E. Nabana and S. I. Pokhozhaev, Nonexistance of global solutions to an elliptic equation with nonlinear dynamical boundary condition, Bol. Soc. Paran. Mat. 22 (2004), 9-16.

[32] J.-L. Lions, Quelques Méthodes de Résolutions des Problèmes aux Limites Non Linéaires, Dunod, Paris (1969).

[33] J. L. Vázquez and E. Vitillaro, Heat equation with dynamical boundary conditions of reactive type, Commun. Partial Differ. Equations 33 (2008), 561-612.

[34] J. L. Vázquez and E. Vitillaro, Heat equation with dynamical boundary conditions of locally reactive type, Semigroup Forum 74 (2007), 1-40.

[35] E. Vitillaro, Global existence for the heat equation with nonlinear dynamical boundary conditions, Proc. R. Soc. Edinb., Sect. A, Math. 135 (2005), 175-207.

[36] E. Vitillaro, On the Laplace equation with non-linear dynamical boundary conditions. Proc. Lond. Math. Soc. 93 (2006), 418-446.

[37] Z. Yin, Global existence for elliptic equations with dynamic boundary conditions, Arch. Math. 81 (2003), 567-574. 